\title{
Second to Fourth Digit Ratio in Patients with Hirsutism and Its Correlation with Hormonal Assay
}

A.A.Eltaweel ${ }^{1}$, A.M.Hamed ${ }^{2}$, E.M.El-Sebaey ${ }^{3}$ and D.M.Noor ${ }^{4}$

${ }^{1}$ Professor of Dermatology, Venereology and Andrology Dept., Faculty of Medicine, Benha Univ., Benha, Egypt

${ }^{2}$ Assistant Professor of Dermatology, Venereology and Andrology Dept., Faculty of Medicine, Benha Univ., Benha, Egypt

${ }^{3}$ Assistant Professor of Clinical and Chemical Pathology Dept., Faculty of Medicine, Benha Univ., Benha, Egypt

${ }^{4}$ (M.B.B.CH), Faculty of Medicine, Ain shams Univ., Cairo, Egypt

E-Mail: dinamagdyderma@gmail.com

\begin{abstract}
Hirsutism will be a basic clinical state seen On female patients of constantly on ages. It influences around 5-10\% of the ladies. The cause may be mostly hyperandrogenism, which might be ovarian or adrenal. It might be and only an uncommon metabolic syndrome, medication induced, alternately barely idiopathic infection. The point about this fill in may be with assess those seconds should fourth digit length proportion over patients with hirsutism and its correspondence with hormonal profile. This examine included 50 female patients torment from hirsutism as stated by altered Ferriman Furthermore Gallwey score, done addition, 30 Obviously solid people from claiming matched age. The results of the present study showed that patients had statistically significant higher levels than controls in Prolactin $[\mathrm{P}=0.000]$ and DHEA-S $[\mathrm{P}=0.005]$ with no statistically significant difference in other hormones and there was no significant correlation between second to fourth digit ratio and all the laboratory investigations in both hands. the study concluded that the assessment of hirsutism is a multifactorial and that 2D:4D ratio is very promising tool in the diagnosis of hirsutism and the assessment of its severity.
\end{abstract}

Keywords: Hirsutism, 2D, 4D, Hormonal, Cross-Sectional.

\section{Introduction}

Hirsutism will be a basic clinical state seen On female patients of constantly on ages. It influences around 5-10\% of the ladies What's more will be An basic protestation in the dermatological outpatient Branch for cosmea motivations. The cause may be mostly hyperandrogenism, which might be ovarian or adrenal. It might be and only an uncommon metabolic syndrome, medication induced, alternately barely idiopathic infection [1].

The symptomatic assessment for hirsutism by includes two steps. In those vicinity for hirsutism must make affirmed Eventually Tom's perusing immediate examination of the patients, Concerning illustration huge numbers people with unwanted hair don't really bring terminal hair development done a male-like design What's more this will make identifier with Ferriman Also Gallwey scoring system; second, copartnered alternately etiological abnormalities Also issue must make excluded through investigations, those essential examination altogether ladies for hirsutism about whatever seriousness may be An plasma downright testosterone and the $\mathrm{r}$ will be will avoid states which might result in hirsutism Similarly as measuring dehydroepiandrosterone sulfide [DHEA-sulfate] which will be Exceptionally raised to androecium secreting tumors, Furthermore measuring 17 hydroxyprogesterone to screening patients to non-classic intrinsic adrenal hyperplasia [2] Furthermore measuring serum prolactin should avoid hyperprolactinemia [3].

The reason for hirsutism might be separated into, nonandrogenic factors, androecium abundance Furthermore idiopathic infection hirsutism [4]. Non-androgenic reason for hirsutism would moderately rare, androgenetic reasons would beyond question the the vast majority basic reason for hirsutism, representing more or less $75-85 \%$ for such patients. Androecium issue include: PCOS, which influences around $70-80 \%$ about hirsute women, hyperandrogenic insulin-resistant acanthosis nigricans syndrome, influencing regarding 3\%, 21-OH-deficient non-classic adrenal hyperplasia On $2-8 \%$ of patients and, rarely, ovarian or adrenal androgen-secreting neoplasms [5].

Digit proportion is the proportion from claiming length of separate digits alternately fingers commonly measured from those mid-point of lowest part fold the place the fingers join the hands of the tip of the fingers. However, the greater part commonly, digit proportions demonstrate best 2D [index finger]:4D [ring finger] proportions [6]. Those proportion of the second digit [index finger $=2 \mathrm{D}$ ] of the fourth digit [ring digit $=4 \mathrm{D}$ ] length need been guessed will reflect prenatal androecium introduction and the individual's affectability to androgens [7]. In females those $2 \mathrm{D}$ is as a rule more drawn out over 4D. This sexual orientation distinction in digit period proportions need been connected for the in-utero parity from claiming androecium will estrogen. It need been suggested that the 2D:4D proportion may be associated negatively with prenatal testosterone levels [8].

Confirmation over help about this see hails from those vicinity about mascot alternately easier digit proportions for Youngsters with intrinsic adrenal hyperplasia [CAH] who have higher androecium presentation Furthermore females suffice from $\mathrm{CAH}$ and also blacks [9]. Likewise, over Klinefelter's syndrome, higher or ladylike digit ratios, attributable should a low testosterone introduction have been accounted for [6]. Couple investigations need accounted for that maternal smoking auto Throughout pregnancy elevates those prenatal testosterones What's more thus is connected with low or mascot 2D:4D proportions in the know youngsters [10].

Will help this hypothesis, regulate associations have been indicated the middle of fetal testosterone What's more 2D:4D Toward dissecting those amniotic hole [11]. A significant number of investigations need assessed the putative effect of prenatal androgens ahead physical, mental Also restorative aspects by utilizing those 2D:4D proportion [12]. However, the relationship between 
hirsutism and the 2D:4D ratio has not yet been investigated.

This study was conducted as a case control study that involved 50 female patients with hirsutism and 30 healthy, age and sex matched controls between the age of 18 and 65 years recruited from Dermatology outpatient clinic, Benha University Hospital. All details of the study were discussed with the patients and informed consent was obtained from each patient before enrolment in the study. The protocol was revised and approved by the Ethics Committee of Human Research., Faculty of Medicine, Benha University.

Patients with any of the following conditions were excluded from the study: autoimmune

\section{Material and methods}

This study was conducted as a case control study that involved 50 female patients suffering from hirsutism according to modified Ferriman and Gallwey score, in addition, 30 apparently healthy individuals of matched age and sex was chosen as a control group.

Patients who were excluded from the study:

1. Patients below the age of 18 years or above the age of 45 years.

2. Patients on corticosteroids and oral contraceptives during the last six months before enrolment in the study.

3. Patients with musculoskeletal deformities in the hand.

4. Pregnant and lactating females.

5. Patients with known thyroid illness or peripheral ischemia which may affect hair distribution.

\subsection{History taking}

Patients and controls were subjected to full history taking: Including personal history, family history, as well as history of other skin diseases or drug intake.

\subsection{Clinical examination}

Clinical examination was mainly done through modified Ferriman and Gallwey scale for hirsutism as a score of 1 to 4 is given for nine areas of the body [Ferriman and gallwey, 1961]. Score for normal is from 0 to less than 8 , scores from 8 to 15 is considered mild hirsutism, score more than 15 is considered moderate or severe hirsutism.

\subsection{Laboratory investigation}

$5 \mathrm{ml}$ of venous blood was taken by venipuncture from the subjects into dry plain tube and left at room temperature for about $20 \mathrm{~min}$ to be clotted then centrifuged at $1300 \mathrm{rpm}$. The serum was separated and divided in 2 aliquots and stored in freezer at $-20{ }^{\circ} \mathrm{C}$ till analyzed .

Determination of:

- Follicle Stimulating Hormone [FSH]

- Luteinizing Hormone [LH]

- Serum Testosterone

- Dehydroepiandrosterone-sulfate [DHEA-sulfate].

- Serum Prolactin

- 17 Hydroxy Progesterone.

\subsection{Statistical Analysis}

Data were collected, revised, coded and entered to the Statistical Package for Social Science [IBM SPSS] version 23. The quantitative data were presented as mean, standard deviations and ranges when their distribution found parametric and median with inter-quartile range [IQR] when their distribution found non-parametric. Also, qualitative variables were presented as number and percentages.

\section{Results and discussion \\ 3.1 Clinical results}

The current study was carried out on 50 female patients with hirsutism their ages ranged from 20 to 40 years with a mean age of $30.35 \pm 6.02$ years. The control group included 30 healthy, age-matched women. Their ages ranged from 20 to 42 years with a mean age of $28.93 \pm$ 6.20years. No statistically significant differences were found between patients and controls regarding age Table (1). In all telogen effluvium patients, the hair pull test was positive all over the scalp [including frontal, 2 temporal and occipital regions].

Clinical Data of patients with hirsutism: Twenty-two [44\%] patients reported to have positive family history of hirsutism while most of the patients had no history of PCOS Table (2).

Table (1) Comparison between patients and controls regarding age

\begin{tabular}{lccccc}
\hline Age & Patients group & Control group & Test value $^{*}$ & P-value & Sig. \\
\hline Mean \pm SD & $30.35 \pm 6.02$ & $28.93 \pm 6.20$ & 1.007 & 0.317 & NS \\
Range & $20-40$ & $20-42$ & & \\
\hline \multicolumn{5}{c}{ *: Independent t-test }
\end{tabular}

Table (2) Prevalence of PCOS and Family history in patients' group

\begin{tabular}{llll}
\hline & & No. & $\%$ \\
\hline \multirow{2}{*}{ PCOS } & Negative & 38 & $76.0 \%$ \\
& Positive & 12 & $24.0 \%$ \\
\multirow{2}{*}{ Family History of Hirsutism } & Negative & 28 & $56.0 \%$ \\
& Positive & 22 & $44.0 \%$ \\
\hline
\end{tabular}


As regard modified Ferriman \&Gallwey [mFG] score, statistically significant increase was found in patients than in controls $[\mathrm{P}=$ less than 0.001$]$ Table (3)

Table (3) Comparison between patients and controls regarding mFG score

\begin{tabular}{|c|c|c|c|c|c|}
\hline F\&G score & $\begin{array}{c}\text { Patients group } \\
\text { No. }=\mathbf{5 0}\end{array}$ & $\begin{array}{c}\text { Control group } \\
\text { No. }=\mathbf{3 0}\end{array}$ & Test value* & P-value & Sig. \\
\hline $\begin{array}{l}\text { Mean } \pm \text { SD } \\
\text { Range }\end{array}$ & $\begin{array}{c}15.88 \pm 4.45 \\
9-28\end{array}$ & $\begin{array}{c}6.17 \pm 0.79 \\
5-7\end{array}$ & 11.821 & $<0.001$ & $\mathrm{~S}$ \\
\hline
\end{tabular}

According to the severity of hirsutism, twenty-six [52\%] of the hirsutism patients had mild hirsutism and twenty-four [48\%] had moderate to severe hirsutism Table (4)

Table (4) Severity distribution among the studied cases

\begin{tabular}{lll}
\hline Severity & No. & $\%$ \\
\hline Mild & 26 & $52.0 \%$ \\
Moderate to severe & 24 & $48.0 \%$ \\
Total & 50 & $100.0 \%$ \\
\hline
\end{tabular}

When Comparing between patients and the controls regarding 2D, 4D and their ratio in the both hands, 2D to 4D ratio was significantly increased in right hand $[\mathrm{P}=0.000]$ and also in the left hand $[\mathrm{P}=0.000]$ in hirsutism patients than controls Table (5)

Table (5) Comparison between patients and controls regarding 2D, 4D and their ratio in both hands

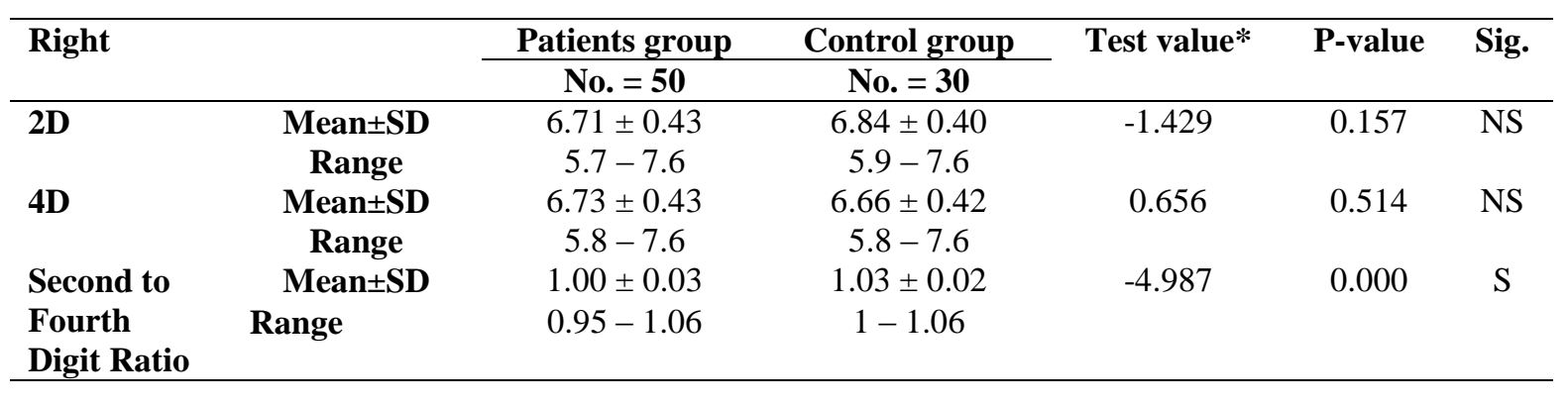

$*$ : Independent t-test
Relation between 2D to 4D Ratio and Clinical Data

No statistically significant relation was found between 2D:4D ratio and PCOS in both right hand [P=0.544] and left hand $[\mathrm{P}=0.689]$ Table (6).

Table (6) Correlation between second to fourth digit ratio of both hands with PCOS

\begin{tabular}{lcccccc}
\hline & & Negative PCOS & Positive PCOS & \multirow{2}{*}{ Test value } & \multirow{2}{*}{ P-value } & \multirow{2}{*}{ Sig. } \\
\cline { 3 - 4 } & & No. $=\mathbf{3 8}$ & $\mathbf{N o .}=\mathbf{1 2}$ & & \multirow{2}{*}{0.611} & \multirow{2}{*}{0.544} \\
\cline { 3 - 4 } Second to Fourth & Mean \pm SD & $1.00 \pm 0.03$ & $0.99 \pm 0.03$ & & \multirow{2}{*}{ NS } \\
Digit Ratio [right] & Range & $0.95-1.06$ & $0.97-1.05$ & & \multirow{2}{*}{ NS } \\
Second to Fourth & Mean \pm SD & $1.00 \pm 0.02$ & $1.00 \pm 0.03$ & \multirow{2}{*}{0.403} & 0.689 \\
Digit Ratio [left] & Range & $0.97-1.06$ & $0.97-1.07$ & & &
\end{tabular}

*: Independent t-test

\subsection{Discussion}

Hair follicles in the human embryo principal happen during more or less 9 weeks about gestation, for practically Creating Eventually Tom's perusing week 16, harmonizing nearly for the top of prenatal androgens and the determination about digit proportions [13] .

Those point for this consider might have been on evaluate those second on fourth digit period proportion 
[2D:4D] Previously, patients with hirsutism Also its correspondence for hormonal profile. This might have been done through assessment about 50 patients suffice starting with hirsutism as stated by changed Ferriman Furthermore Gallwey [mFG] score What's more 30 clinically allowed members served Likewise a control one assembly.

Following taking point by point history Also looking at both patients and controls Furthermore settling on the necessary lab investigations for the analysis of the reason for hirsutism, it might have been watched that hirsutism patients required statistically huge higher levels over controls done Prolactin $[\mathrm{P}=0$. 000] and DHEA-S $[\mathrm{P}=0$. 005], At there might have been no noteworthiness to whatever remains of the investigations which were downright testosterone [TT], follicle fortifying hormone $[\mathrm{FSH}]$, luteinizing hormone $[\mathrm{LH}]$ and 17 hydroxyprogesterone.

This might have been in understanding J. Lakhani Som [14] who expressed that, expanded prolactin might have been display On 21. $05 \%$ from claiming patients for hirsutism and the DHEAS levels were raised On 11. 36\% from claiming patients for hirsutism, and S.T. Tabari [15] who affirmed the vitality of hyperprolactinemia Concerning illustration a standout amongst the reason for hirsutism, Likewise hyperprolactinemia might have been Exceptionally predominant Around hirsutism patients.

V. Krishnendra [16] also accounted that testosterone level might have been raised Previously, 43. 3\% Furthermore might have been typical On 56. $7 \%$ about patients. LH/FSH proportion might have been raised to 46 . 7\% same time ordinary clinched alongside 53. $3 \%$ from claiming patients.

As opposed with this study, A.K. Khalaf [17] found no huge distinction for serum levels from claiming prolactin recognized between hirsutism patients and control gatherings What's more [16] reasoned that hormonal lopsidedness will be seen over patients displaying for hirsutism Also hormones similar to testosterone might have been raised clinched alongside $43.3 \%$ about patients, LH: fsh might have been brought up Previously, 46. 7\% from claiming patients .

Those distinctive outcomes might underscore that the pathogenesis of hirsutism will be multifactorial Furthermore vague Likewise hirsutism comes about starting with an interactional the middle of androecium levels Furthermore hair follicle affectability with androecium [18].

This might be clarified Likewise [19] finished up that almost every one hirsute ladies need an expanded in androgens, generally testosterone, yet the build might not be addition on raise those serum aggregate testosterone focus over those ordinary reach a direct result those transporter protein to testosterone, sex hormone-binding globulin, may be stifled At androecium preparation will be expanded. In the remaining women, those hirsutism might be expected on expanded change from claiming testosterone on dihydrotestosterone Toward those catalyst $5 \alpha$-reductase clinched alongside fringe tissue, including hair follicles, this metabolite may be more intense over testosterone Thus, raised $5 \alpha$-reductase movement need been exhibited in the hair follicles for ladies with idiopathic infection hirsutism, What's more overabundance hair Growth will be prone will be expected should an overstated reaction of the hair follicle should ordinary androecium levels .

Previously, our study, twenty-two [44\%] of the patients required a sure family history in the first-degree relatives As far as torment for hirsutism and twenty-eight [56\%] of the patients required negative family history .

In distinctive studies, distinctive percent have been accounted for As far as certain gang record Around individuals with hirsutism. A certain gang record from claiming hirsutism might have been discovered Previously, 42. $5 \%$ of patients On contemplate by [20], $24.1 \%$ of patients in study by [21], $50 \%$ in study by [22], $40.7 \%$ in study by [23] and $29 \%$ of patients in study [24].

In the present study, throughout historical backdrop taking, 24\% from claiming hirsutism patients offered historical backdrop for PCOS. Different investigations bring accounted distinctive percent As far as sure history of PCOS [14] accounted for that $46 \%$ from claiming hirsutism patients satisfied the criteria to PCOS, [25] evaluated those PCOS pervasiveness to a chance to be $13 \%$ Previously, hirsutism patients Furthermore [26] found $47 \%$ from claiming hirsutism patients were diagnosed for PCOS.

The Contrast Might be clarified as, in our investigation PCOS might have been known through the history of the patients Also that might have been not sufficient for those symptomatic criteria of the PCOS and its full investigations Similarly as ultrasonography will be imperative in the analysis of PCOS .

In the current study, the proportion the middle of the second and the fourth digit length [2D:4D ratio] might have been altogether easier clinched alongside hirsutism patients over in controls On both control $[\mathrm{P}=0.000]$.

In spite of the fact that those association the middle of hirsutism Also 2D:4D proportions might have been not investigated before, 2D:4D required been examined clinched alongside huge numbers illnesses that are influenced Eventually Tom's perusing androecium overabundance or expanded androecium receptors affectability.

This originated in understanding later on when [27] stated that low, that's only the tip of the iceberg male type, 2D:4D proportions were connected with deferred selfreported menarche Furthermore [28] underpinned that those 2D: 4D proportion may be a predictor for skin break out development, duration, What's more seriousness grade, no less than On female patients.

Also, Ö. Bilgiç [29] discovered that an easier 2D:4D proportion in the right hand is connected with aga Previously, guys for a gentle to direct impact measure and this might have been after the fact suitably for [30] who inferred that those left hand digit proportion 2D:4D is easier in aga patients, and therefore, left hand digit proportion 2D:4D could be utilized Concerning illustration a estimation device around for aga improvement. 
D. Bola [31] inferred that there may be a association the middle of digit proportion and deep rooted premature discharge also, [32] noted that those 2D:4D proportion could be utilized as an instrument for right on time prediction from claiming PCOS.

Likewise respect to our lab investigations when associated with 2D:4D proportion the bring about our investigation demonstrated non-significant relationship the middle of second to fourth digit proportion Also every last one of lab investigations On both control. This went in understanding with [33] who found non-significant acquaintanceships between 2D:4D and testosterone, oestradiol, the proportion from claiming testosterone to oestradiol, progesterone, luteinizing hormone, follicle empowering hormone, alternately inhibin b over Possibly guys alternately females , [34] affirmed that there might have been non noteworthy relationship the middle of digit proportion variables Furthermore circle hormone levels about progesterone, testosterone Furthermore [35] also stated that to both guys What's more females, there were no huge correlations the middle of digit proportion What's more At whatever androecium alternately estrogen focuses.

As opposed on these results, A.K. Trojan [36], found that 2D:4D over men for gynecomastia might have been essentially diminished over the proportion for control men [p $<0.0001]$ Also this indicates the correspondence the middle of 2D:4D Also prolactin level. T.Mitsui [37] expressed that 2D:4D might have been negatively associated with DHEA in guys main $[p<0$. 01] Yet no correlations were watched in the different adrenal steroid hormones tried to guys or clinched alongside At whatever adrenal steroid hormones On females.

This might make clarified Concerning illustration [38] expressed that the connections the middle of 2D:4D Furthermore levels about sex steroids to mature people need aid lesquerella clear, Likewise a lot of people correlational investigations about 2D:4D Also grown-up sex steroids bring reasoned that this Acquaintanceship is statistically non-significant same time 2D:4D digit proportion recognized to make An biomarker of the offset between fetal testosterone [T] What's more estrogen [E].

The hirsutism might a chance to be not due with increment of the serum androecium yet all the because of the expanded transformation for testosterone with dihydrotestosterone by those catalyst $5 \alpha$-reductase for fringe tissue, including hair follicles. Thus, raised $5 \alpha$ reductase action need been showed in the hair follicles for ladies with idiopathic infection hirsutism, Furthermore overabundance hair Growth will be likely will be expected with a misrepresented reaction of the hair follicle will typical androecium levels.

S. Taheri [40] reasoned that done patients with idiopathic infection hirsutism $[\mathrm{IH}]$, a expansion in the nearby add up about dynamic androgens were observed, which Might that point a chance to be utilized Similarly as An substrate to other androecium metabolic routes.

In the current study, there was non-statistically significant there might have been non statistically critical Contrast over 2D:4D between those hirsutism patients for
PCOS and hirsutism patients without PCOS for both right hand $[\mathrm{P}=0.544]$ and left hand $[\mathrm{P}=0.689]$, this might have been in understanding with [41] who expressed that ladies with PCOS don't exhibit finger period examples that would steady for expanded prenatal androecium introduction Be that these discoveries don't preclude An part to prenatal androgens in the improvement from claiming PCOS.

As opposed on this study, [42] closed that ladies with PCOS might have masculinized finger length examples with low 2D:4D steady for expanded prenatal androecium exposure, Also , [32] stated that there might have been statistically critical distinction between 2D:4D about PCOS instances and controls also others 2D:4D could be utilized Likewise an instrument to right on time prediction about polycystic ovarian syndrome [PCOS].

Comparative brings about distinctive states were news person Eventually Tom's perusing [28] who stated that skin break out seriousness might have been emphatically corresponded with those imply 2D: 4D ratio, and [43] inferred that 2D:4D proportion might have been were differentially identified with the seriousness about premenstrual side effects On solid youthful ladies. Low straight 2D:4D Also helter skelter exited 2D:4D were positively connected with secondary seriousness manifestation scores.

\section{Conclusion}

Those objective for surveying hirsutism is should endeavor to determine those particular etiology and this must a chance to be carried out through kept tabs history.

\section{References}

[1] S. Sachdeva , Hirsutism: Evaluation and Treatment. Indian J Dermatol.Vol. 55(1), PP.3-7,2010.

[2] S.Page and N.Hewapathirana ,Hirsutism Investigations, diagnosis and treatment. Rila Publications Ltd. Clinical Focus Primary Care. Vol. 7(1) ,PP. 16-2416,2013.

[3] R.S. Legro, S.A. Arslanian, D.A.; Ehrmann, K.M. Hoeger, M.H. Murad and R. Pasquali, Diagnosis and treatment of polycystic ovary syndrome: an endocrine society clinical practice guideline. J Clin Endocrinol Metab. Vol.98(12),PP.4565-92,2013.

[4] J.K. Zawadzki and A. Dunaif ,Diagnostic criteria for polycystic ovary syndrome: towards a rational approach. In: Dunaif A, Givens JR, Haseltine FP, Merriam GR, editors. Polycystic Ovary Syndrome. Boston: Blackwell Scientific Publications. Vol.1 ,PP.377-384,1992.

[5] N.K. Agrawal , Management of Hirsutism. Indian J Endocrinal Metab. Vol. 17(Supp1) ,PP. SS7-S82 ,2013.

[6] S. Jeevanandam and P.K. Muthu ,2D:4D Ratio and its Implications in Medicine. J Clin Diagn Res. Vol. 10(12) ,PP. CM01-CM03 2016.

[7] J. Hönekopp and S. Watson , Meta-analysis of digit ratio 2D:4D shows greater sex difference in the right hand. Am J Hum Biol. Vol. 22, PP.619-630. ,201 • 
[8] Z. Zheng and M.J. Cohn, Developmental basis of sexually dimorphic digit ratios. Proc Natl Acad Sci. Vol. 108,PP.16289-16294. ,2011.

[9] C. Ciumas, A.L.Hirschberg and I. Savic, High fetal testosterone and sexually dimorphic cerebral networks in females. Cereb Cortex. Vol. 19(5) ,PP.1164$1172,2009$.

[10] S.M. Coyne, J.T. Manning, L.Ringer and L. Bailey , Directional asymmetry [right-left differences] in digit ratio [2D: 4D] predict indirect aggression in women. Personality and Individual Differences. Vol. 43(4) ,PP.865-872,2007.

[11] S. Lutchmaya, S. Baron-Cohen, R. Knickmeyer and J.T. Manning, 2nd to 4th digit ratios, fetal testosterone and estradiol. Early Hum Dev. Vol. 77[12] ,PP.23-8. ,2004.

[12] J.T. Manning, Resolving the role of prenatal sex steroids in the development of digit ratio. Proc Natl Acad Sci. Vol. 108,PP.16143-16144,2011.

[13] O. Bilgic, H.C. Altınyazar, D. Ery1lmaz and Z.A. Tuğrul ,Are 2D:4D finger-length ratios an indicator of androgenetic alopecia in males? An Bras Dermatol. Vol. 91(2) ,PP. 156-159,2016.

[14] J. Lakhani Som, J. Lakhani Om and R.C. Raval, A study of endocrine profile in premenopausal women with hirsutism. J integ health sci. Vol. 2(2),PP.17-22. ,2014.

[15] S.T. Tabari, M. harbatdaran, S.M. Afkham and M. Montazeri, Hyperprolactinemia and Hirsutism in Patients Without Polycystic Ovary Syndrome. Int J Trichology, Vol. 8(3),PP. 130-134. ,2016.

[16] V. Krishnendra and A.S. Singh , A Study of Hormonal profile in Hirsutism patients-A prospective observational study in a Tertiary Care Hospital. IndJ Clinic and Exp Derm. Vol. 2[2], PP.58- 61 ,2016.

[17] A.K. Khalaf and N.A. Naji , Relationship between Sex Hormones and Hirsutism in Women. Diyala $\mathbf{J}$ Med. Vol. 9 (2) ,PP.76-81,2015.

[18] D.K. Song, H.J. Lee, Y.S. Hong and Y.A. Sung , Insulin resistance is associated with hirsutism in unselected reproductive-aged women. Clinic Endo. Vol. 90,PP.44. ,2019.

[19] R. Tahvilian, A. Ebrahimi ,B. Omid , H. Nemati and S. Masoud, Preparation and Clinical evaluation of Finastride gel in the treatment of idiopathic Hirsutism. J Drug Assess. Vol. 4(1), PP.12-18. ,2015.

[20] S. Chhabra , R.K. Gautam, B. Kulshreshtha, A. Prasad and N. Sharma, Hirsutism: A Clinico-investigative Study. Int J Trichology. Vol. 4(4) ,PP. 246-250. ,2012.

[21] A.K. Ghalib and S.S. Tahir, Prevalence of hirsutism in adult females in gynecology outpatient Clinic at Tikrit Teaching Hospital. J Fac Med Baghdad. Vol. 47,PP.44-8. ,2005.

[22] L.A. Brodell and M.G. Mercurio , Hirsutism: diagnosis and management. Gend Med. Vol. 7,PP.7987. ,2010.

[23] Yazdanfar, Z. Beyhaghi and N. Manouchehrian , Frequency of hirsutism in female students of
Hamadan university of medical sciences. J Hamadan Univ Med Sci. Vol. 4,PP.26-32. ,1997.

[24]F. Farnaghi, H. Seyrafi and N. Zarrinpour , Descriptive study of 110 patients with hirsutism in Tehran Razi Hospital during the years 2000-2001. Iran J Dermatol. Vol. 6,PP.21-25. ,2002.

[25] Jalilian, F. Kiani F. Sayehmiri, and M .Akbari , Prevalence of polycystic ovary syndrome and its associated complications in Iranian women: A metaanalysis. Iran J Reprod Med. Vol. 13(10) ,PP. 591604. ,2015.

[26] G.D. Fede, P. Mansueto, I. Pepe, G. BattistaRini and E. Carmina, High prevalence of polycystic ovary syndrome in women with mild hirsutism and no other significant clinical symptoms. Fertility and Sterility. Vol. 94(1),PP.194-197. ,2010.

[27] R.L. Matchock , Low Digit Ratio [2D:4D] is Associated with Delayed Menarche. Am J Hum Biol. Vol. 20(4) ,PP.487-489. ,2008.

[28]Ö. Bilgiç, M. Doğdu, G.K. İslamoğlu and C. Altinyazar, The relationship between the second to fourth digit ratio and acne vulgaris. J Eur Acad Dermatol Venereol. Vol. 28(10) ,PP.1340-1343. ,2013.

[29] Ö. Bilgiç, C. Altınyazar, H. Hıra and M. Doğdu ,PP. Investigation of the association of the second to fourth digit ratio with skin sebum levels in females with acne vulgaris. Am J Clin Dermatol. Vol.16,PP.559-564. , 2015.

[30] M. Unal, , Digit ratio 2D:4D is a possible indicator for androgenetic alopecia in males. J Cosmet Dermatol. Vol. 17(3) ,PP.545-548. ,2017.

[31] D. Bolat, G.U. Kocabas, T. Kose, Degirmenci T, M.E. Aydin and C. Dincel, The relationship between the second-to-fourth digit ratios and lifelong premature ejaculation: a prospective, comparative study. Andrology. Vol. 5(3),PP.535-540. ,2017.

[32] R. Roy, R. Kundua, M. Senguptab and P. Soma , Association between digit length ratio [2D:4D] and polycystic ovarian syndrome [PCOS]—A study among eastern Indian population. J Anat Soc Ind. Vol. 67(2),PP.S14-S19. ,201^.

[33] T. Mitsui, , A Araki, S. Imai Sato, and C. Miyashita, Effects of prenatal Leydig cell function on the ratio of the second to fourth digit lengths in school-aged children. PLoS One. Vol. 10 (3) ,PP. e0120636. ,2015.

[34] G. Richards, M. Klimek, G. Jasienska and U.M. Marcinkowska ,Digit ratio [2D:4D] and circulating testosterone, oestradiol, and progesterone levels across the menstrual cycle. Ear Hum Dev. Vol. 117,PP. 6873. 2018.

[35] L.P. Hollier, J.A. Keelan, E.S. Jamnadassa, M.T. Maybery and A.J. Whitehouse, Adult digit ratio [2D:4D] is not related to umbilical cord androgen or estrogen concentrations, their ratios or net bioactivity. Earl Hum Dev. Vol. 91(2) ,PP.111-117. ,201.

[36] A.K. Trojan and B. Antoszewski , Can digit ratio [2D:4D] studies be helpful in explaining the aetiology of idiopathic gynecomastia? Early Hum Dev. Vol. 91(1) ,PP.57-61. ,2010. 
[37] T.Mitsui ,A. Araki ,H. Goudarzi, and S.Sasaki , Effects of adrenal androgens during the prenatal period on the second to fourth digit ratio in schoolaged children. J Steroids. Vol. 113,PP. 46-51,2016

[38] J. Manning, L. Kilduff, and B. Fink , Digit ratio [2D:4D]: a biomarker for prenatal sex steroids and adult sex steroids in challenge situations. Front Endo [Lausanne], Vol. 5,PP.9. ,2014.

[39] R. Tahvilian, A. Ebrahimi, B. Omid, H. Nemati and S. Masoud, Preparation and Clinical evaluation of Finastride gel in the treatment of idiopathic Hirsutism. J Drug Assess. Vol. 4(1),PP.12-18. ,2015.

[40] S. Taheri, G. Zararsiz, S. Karaburgu, M., Borlu and M.T. Ozgun, Is idiopathic hirsutism [IH] really idiopathic? mRNA expressions of skin steroidogenic enzymes in women with IH. Euro J Endo. Vol. 173[4] ,PP.447-454. ,2015.

[41] M.E. Lujan, T.G. Bloski, D.R. Chizen, D.C. Lehotay and R.A. Pierson, Digit ratios do not serve as anatomical evidence of prenatal androgen exposure in clinical phenotypes of polycystic ovary syndrome. Hum Rep. Vol. 25(1) ,PP. 204-211. ,2010.

[42] V.K. Pandit, M. Setiya, and M .Jehan, Digit ratio [2D:4D]: A Potential Anatomical Biomarker for Predicting the risk of development of Polycystic Ovarian Syndrome. IOSR Journal of Dental and Medical Sciences. Vol. 15(08) ,PP.58-64. ,2016.

[43] Y.Kaneoke and Shimokawa T , Severity of Premenstrual Symptoms Predicted by Second to Fourth Digit Ratio. Front. Med. Vol. 4,PP.144. ,2017. 